\title{
Limited ankle dorsiflexion increases the risk for mid-portion Achilles tendinopathy in infantry recruits: a prospective cohort study
}

\author{
Alon Rabin $^{1 *}$, Zvi Kozol $^{1}$ and Aharon S Finestone $2,3,4$
}

\begin{abstract}
Background: Achilles tendinopathy (AT) is a prevalent condition among runners and military personnel. Although ankle dorsiflexion (DF) range of motion (ROM) as measured with the knee bent has not been previously associated with AT, the literature concerning its role is limited. In addition, the role of lower extremity movement pattern in the pathogenesis of AT has not been studied prospectively.

The purpose of this study was to further explore the role of ankle DF ROM as measured with the knee bent and that of lower extremity movement pattern as risk factors for mid-portion AT.

Methods: Seventy healthy male military recruits (mean \pm SD age, height and body mass of $19.6 \pm 1.0$ years, $176.0 \pm 10.0 \mathrm{~cm}$, and $71.5 \pm 7.4 \mathrm{~kg}$ ) participated in this study. Ankle DF ROM as measured with the knee bent in weight-bearing (WB) and non-weight-bearing (NWB), as well as lower extremity quality of movement during a lateral step down (LSD) test were measured at baseline. Participants were then followed for a 6-month period of army basic training with recording of the development of AT.
\end{abstract}

Results: Five participants developed AT during training. Participants that developed AT had a more limited NWB ankle DF ROM $\left(27.4^{0}\right.$ versus $\left.21.1^{\circ}, p=0.025\right)$. The quality of lower extremity movement did not differ between injured and uninjured participants $(p=0.361)$.

Conclusions: A more limited ankle DF ROM as measured in NWB with the knee bent increases the risk of developing AT among military recruits taking part in intensive physical training.

Keywords: Achilles, Tendinopathy, Risk, Ankle, Dorsiflexion

\section{Background}

Achilles tendinopathy (AT) is a common injury among runners and military personnel [1-7]. The disorder is typically classified as insertional, affecting the tendon at its insertion onto the calcaneus, or as mid-portion, affecting the tendon between 2 to $6 \mathrm{~cm}$ proximal to its insertion [8]. This paper will focus on the latter of these 2 entities.

Despite its frequency, only few prospective investigations into the risk factors for AT have been performed. Risk factors are typically classified as intrinsic, or extrinsic. Intrinsic risk factors that have been previously associated with AT include increased age, increased or

\footnotetext{
* Correspondence: alonrabin@gmail.com

'Department of Physiotherapy, Ariel University, Ariel, Israel

Full list of author information is available at the end of the article
}

decreased gastrocnemius flexibility, decreased subtalar motion, increased pronation, and decreased plantar flexor strength $[8,9]$.

Although the flexibility of the gastrocnemius has been previously associated with AT $[10,11]$, the kinematics of running suggest it is the flexibility of the soleus that may be more relevant to the pathogenesis of this condition. While flexibility of the gastrocnemius is assessed by measuring ankle dorsiflexion (DF) with the knee extended, this alignment is never reached during running [12]. Instead, maximal ankle DF is reached during the mid-stance of the running gait cycle, when the knee is flexed approximately $40^{\circ}[12,13]$. The simultaneous ankle DF and knee flexion that occur during the first half of the running cycle, are controlled eccentrically by the ankle plantarflexors and serve to absorb the impact 
of the upper body $[12,14]$. This shock-absorbing mechanism is likely to strain the soleus more than the gastrocnemius as its origin on the tibia makes it suitable for controlling knee flexion under weight-bearing conditions. In fact, it has been previously shown that during running the contribution of the soleus to power absorption increases as compared with walking [15]. Therefore, a measurement of ankle DF with the knee bent, which reflects soleus flexibility, may be more indicative of the functional demands during running. Furthermore, as it has been shown that a more limited flexibility of the gastrocnemius increases absorption work by the plantarflexors during walking [16], it is possible, that a more limited flexibility of the soleus would likewise increase absorption work by the plantarflexors during running, which may lead to increased strain on the Achilles tendon.

Although when previously tested, bent-knee ankle DF has not been associated with AT, findings are limited by few prospective studies $[10,11]$. Therefore, further investigation of this association seems warranted.

Another possible risk factor for AT is an altered lower extremity movement pattern [17]. AT has been associated with excessive foot pronation during running [8], as well as greater hip adduction and knee internal rotation during a leaping maneuver [17]. These kinematic alterations are part of a more general movement pattern involving a medial collapse of the knee which is sometimes referred to as "dynamic knee valgus" [18,19]. Dynamic knee valgus which has been associated with several other lower extremity pathologies [20,21], may also increase the risk for AT.

The current gold standard for assessing lower extremity movement pattern is 3-dimensional motion analysis. However, due to its cost, unique set-up, length of application and required training, this tool may not be feasible in the clinical setting. If a more clinically feasible assessment tool can successfully identify individuals at risk for developing AT this may constitute significant progress in risk assessment, and possibly in injury prevention. The lateral step down (LSD) is a reliable test often used in clinical practice to assess lower extremity movement pattern [22-24]. However, the predictive validity of the LSD in determining the risk for AT, has yet to be determined.

The purpose of this study was to examine whether ankle DF as measured with the knee bent, as well as the quality of movement as measured by the LSD can predict the development of AT in male military recruits undergoing army basic training (ABT).

\section{Methods}

The study was approved by the Institutional Review Board of the Israel Defense Forces (approval No. IDF
964-2010), and all participants provided informed consent prior to participation.

\section{Participants}

Seventy male participants with a mean \pm SD age $19.6 \pm$ 1.0 years, height $176.0 \pm 10.0 \mathrm{~cm}$, and body mass $71.5 \pm$

$7.4 \mathrm{~kg}$ were recruited for this study. Participants were military recruits who were thoroughly screened for any musculoskeletal injury/condition prior to beginning a 26-week ABT period. Inclusion criteria were age 18 years or older and no current complaint of pain in the lower extremities or lumbar spine. Participants were excluded if they could not perform any of the measurements included in the study due to pain, imbalance, or any other limitation.

\section{Examiners}

Four examiners performed data collection for this investigation. Two examiners were physical therapists. One physical therapist, who had 15 years of clinical experience in the management of musculoskeletal conditions, performed all DF ROM measurements. The other physical therapist, with over 25 years of teaching and clinical experience in the field of kinesiology and neurological rehabilitation, performed all LSD assessments. These physical therapists have previously demonstrated a moderate inter-rater reliability when performing the LSD (kappa 0.59) [24], and an excellent inter-rater reliability when performing the weight-bearing (WB) and nonweight-bearing (NWB) DF ROM measurements (intraclass correlation coefficient 0.95 and 0.86 , respectively) [24]. Prior to data collection, the physical therapists met for a 4-hour session in order to review each measurement procedure. The 2 other examiners were orthopaedic surgeons. Both surgeons had more than 15 years of experience in foot and ankle surgery among training populations and more than 15 years of experience in research of overuse injuries. The surgeons were responsible for determining the diagnosis of AT during the follow-up period based on pre-determined criteria.

\section{Baseline measurements}

Demographic data including age, height, body mass, and any past Achilles tendon disorder were collected at baseline. Lower extremity quality of movement (LSD test) and ankle DF ROM were subsequently measured. Ankle DF was measured in WB and NWB, as it has been previously suggested that these measurements may be assessing 2 different constructs [25]. All measurements were performed bilaterally.

The LSD test was performed on a $15 \mathrm{~cm}$ step (Reebok International, Canton, MA, USA). Participants stood by the edge of the step. They were instructed to keep their trunk straight, hands on their waist, and bend their knee 
until the contralateral heel touched the floor next to the step. Participants were asked not to put any weight on the heel once it reached the floor. Participants were also asked to try to maintain the knee of the tested leg over the 2nd toe of the ipsilateral foot during the test (a perpendicular black tape was placed along the front of the step from just under the participant's 2nd toe to the floor in order facilitate the assessment by the examiner) (Figure 1). Participants were given 5 practice repetitions before performing 5 consecutive test repetitions. The side tested first was alternated between consecutive participants.

The examiner was positioned 3 meters in front of the participant and scored the test based on a 7-point scale (0 to 6) [23] (Table 1 ). If any one of the movement deviations outlined in Table 1 was observed during any of the repetitions, the participant was considered as having demonstrated that deviation, and the corresponding point value was assigned. A total score of 0 to 1 was classified as "Good" quality of movement (Figure 1), a total score of 2 to 3 was classified as "Moderate" quality of movement (Figure 2), and a total score of 4 or more was classified as "Poor" quality of movement [23]. As only 3 participants demonstrated a "Poor" quality of movement they were grouped together with participants demonstrating a "Moderate" quality of movement for data analysis.

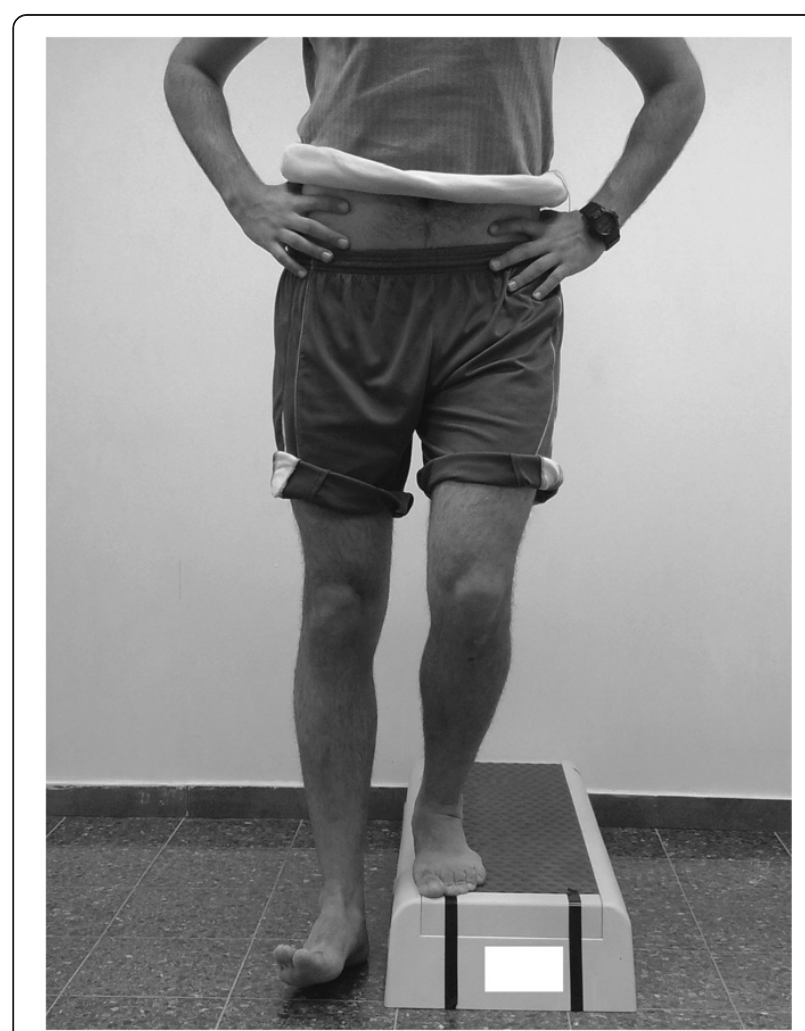

Figure 1 Lateral step down test - good quality of movement.
Table 1 Scoring criteria for the lateral step down test

\begin{tabular}{lll}
\hline Criterion & Interpretation & Score \\
\hline Arm strategy & Removal of a hand off the waist & 1 \\
Trunk alignment & Leaning in any direction & 1 \\
Pelvis plane & Loss of horizontal plane & 1 \\
Knee position & Tibial tuberosity medial to 2nd toe & 1 \\
& $\begin{array}{l}\text { Tibial tuberosity medial to medial } \\
\text { border of foot }\end{array}$ & 2 \\
Steady stance & $\begin{array}{l}\text { Participant stepped down on non } \\
\text { tested limb, or the foot wavered from } \\
\text { side to side }\end{array}$ \\
\hline
\end{tabular}

For the WB DF ROM measurement a $50 \mathrm{~cm}$ long line was marked on the floor and a continuous $60 \mathrm{~cm}$ long line was marked on a wall where the test was to be performed. The participant placed the tested foot along the floor line so that the line bisected the heel and the second toe was on the line. The participant was then asked to lunge forward and bring his patella as close as possible to the vertical line drawn on the wall without lifting the heel off the floor. Once maximal DF was reached the examiner placed an inclinometer (MIE Inclinometer, Nationwide Medical, Inc. Agoura Hills, CA, USA), which was first zeroed on a fixed vertical reference, over the

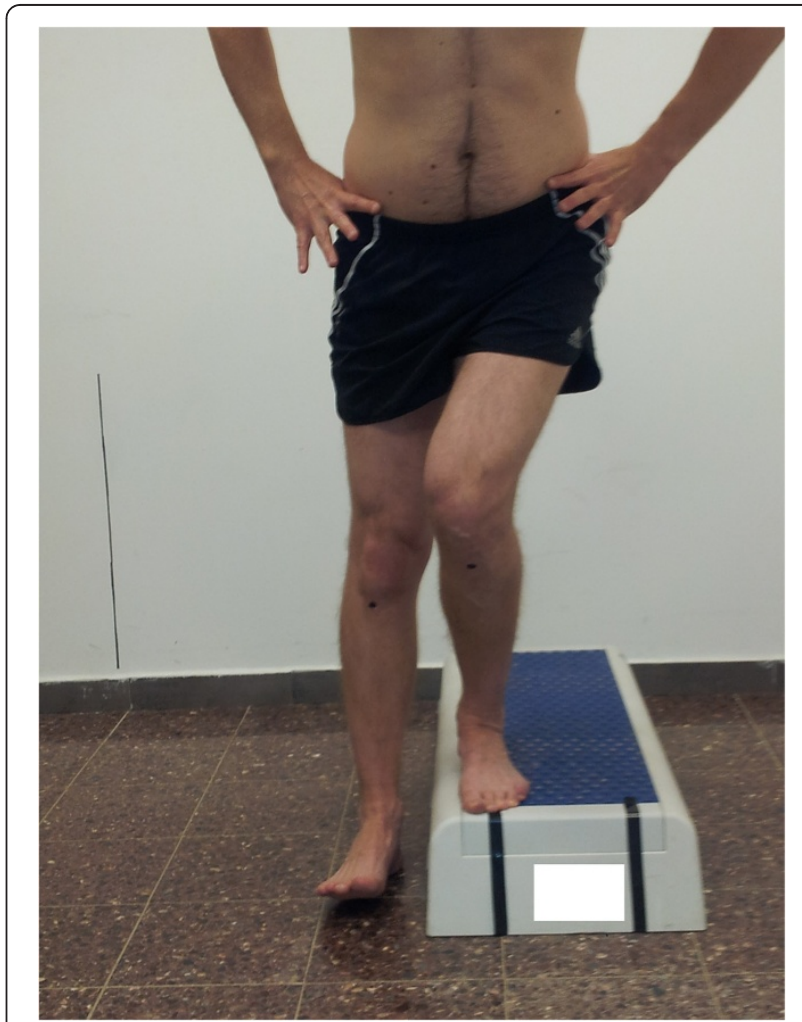

Figure $\mathbf{2}$ Lateral step down test - moderate quality of movement. 
anterior tibia $15 \mathrm{~cm}$ below the tibial tuberosity. The DF angle was recorded and the participant returned to the starting position. The average of 3 measurements was used for data analysis.

For the NWB DF ROM measurement the participant assumed a prone lying position with the knee bent $90^{\circ}$ (Figure 3). The measurement was taken using a universal goniometer (Baseline Plastic Goniometer, The Therapy Connection Inc., Windham, NH, USA). The examiner first manually verified a subtalar neutral position and then leaned his body weight forward over his hands in order to stretch the ankle fully into DF. Dorsiflexion was measured as the angle between the lateral midline of the lower leg (a line from the head of the fibula to the tip of the lateral malleolus) and the lateral border of the foot (a line along the rearfoot/calcaneus). The average of 3 measurements was used for data analysis.

The side tested first, as well as the order of the different DF measurements were alternated between consecutive participants.

\section{Army basic training period}

Participants initiated a 26-week ABT period immediately following baseline measurements. During this period participants went through rigorous physical fitness, as well as military skill training, according to a set and progressively intensified schedule. All participants were exposed to the same training regimen and trained under similar conditions in terms of shoe wear, loads carried, nutrition, and rest periods.

\section{Injury tracking system}

The 2 orthopaedic surgeons met the participants once every 2-3 weeks throughout the course of training. Any complaint was assessed by one of the surgeons with a physical examination, however, no imaging studies were used to verify the diagnosis of AT in this study. A

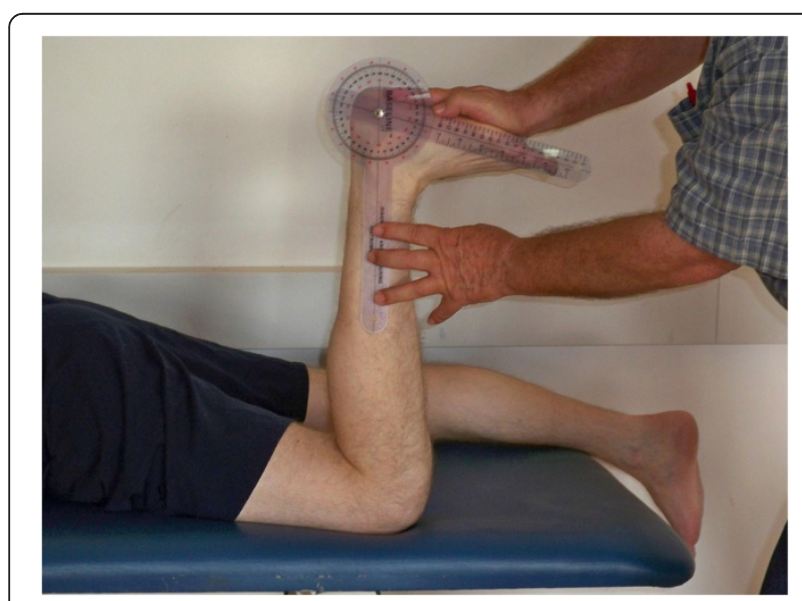

Figure 3 Non-weight-bearing ankle dorsiflexion measurement. participant was considered as having AT if he complained of pain over the tendon during physical activity and presented with tenderness to palpation over the tendon in the area between 2 to $6 \mathrm{~cm}$ proximal to the calcaneal insertion. The participant had to report that palpation over this area reproduced his symptoms. Additional symptoms and signs such as morning stiffness, swelling, warmth, or redness were sought as well, however they were not considered mandatory for establishing the diagnosis.

\section{Statistical analysis}

Baseline measurements were summarized with descriptive statistics using measures of central tendency and dispersion for continuous variables and frequency counts for categorical variables. Univariate associations between the outcome and potential risk factors were analyzed using Mann-Whitney and Fisher Exact tests for interval and categorical variables, respectively. Any variable demonstrating a significant association $(p<0.05)$ was entered into a univariate logistic regression model in order to determine its predictive power. Continuous variables that were significantly associated with the outcome were further analyzed using receiver-operator characteristic (ROC) curves in order to establish their best cutoff value. This cutoff value, which was identified visually as the point nearest the upper left-hand corner of the diagram, corresponds with maximal sensitivity and specificity. All analyses were performed using JMP version 10 statistical package (SAS Institute, Cary, NC) with an a-priori level of significance of $p<0.05$.

\section{Results}

Seventy-five participants were screened. One participant declined participation; another participant was excluded due to suffering an ankle sprain the day before baseline testing, and 2 participants were expelled from ABT for reasons unrelated to their health. A fifth participant died during the follow-up period from septic shock. Thus 70 participants were included in the final analysis. Table 2 presents demographic, history, and physical examination variables of the entire sample, as well as the differences between participants that developed AT and those that did not.

Overall, 5 participants were diagnosed with AT during ABT. All injuries occurred in the right leg. Ankle DF as measured in NWB was more limited among participants that developed AT $(p=0.025)$. Univariate logistic regression also indicated that NWB ankle DF ROM significantly predicted AT $(p=0.007)$. The unit odds ratio (OR) for NWB DF in predicting AT was 0.77 (95\% CI $0.59-0.94)$ which indicates, that for every 1-degree of increased NWB DF ROM, the odds of developing a future AT reduced by 0.23 . The ROC curve for NWB DF 
Table 2 Demographic, history and clinical variables among the sample

\begin{tabular}{|c|c|c|c|c|}
\hline Variable & All participants $(\mathrm{N}=70)$ & Injured $(\mathrm{N}=5)$ & Non-injured $(\mathrm{N}=65)$ & $P$-value \\
\hline Age, $y^{a}$ & $19.6 \pm 1.0$ & $19.7 \pm 0.8$ & $19.6 \pm 1.0$ & 0.661 \\
\hline $\mathrm{BMI}^{\mathrm{a}}$ & $23.1 \pm 2.1$ & $23.2 \pm 2.8$ & $23.1 \pm 2.0$ & 0.965 \\
\hline Past AT, n (\%) yes ${ }^{\text {b }}$ & $0(0.0)$ & $0(0.0)$ & $0(0.0)$ & NA \\
\hline Right WB DF, ${ }^{0}$ a & $49.4 \pm 6.7$ & $43.3 \pm 9.1$ & $49.9 \pm 6.3$ & 0.184 \\
\hline Left WB DF, ${ }^{0}$ a & $56.4 \pm 6.8$ & $50.2 \pm 5.8$ & $56.8 \pm 6.7$ & 0.025 \\
\hline Right NWB DF, 0 a & $27.4 \pm 5.9$ & $21.1 \pm 6.1$ & $27.9 \pm 5.6$ & 0.025 \\
\hline Left NWB DF, ${ }^{0}$ a & $27.3 \pm 6.3$ & $21.3 \pm 7.3$ & $27.8 \pm 6.0$ & 0.067 \\
\hline Right LSD, n (\%) good (score 0 to 1 ) & $33(47.1)$ & $1(20.0)$ & $32(49.2)$ & 0.361 \\
\hline Left LSD, $\mathrm{n}(\%)$ good (score 0 to 1 ) & $33(47.1)$ & $1(20.0)$ & $32(49.2)$ & 0.361 \\
\hline
\end{tabular}

${ }^{a}$ Values are Average \pm SD.

${ }^{\mathrm{b}}$ No statistics are computed for this parameter as it is constant.

Abbreviations: AT Achilles Tendinopathy, BMI Body Mass Index, DF Dorsiflexion, LSD Lateral Step Down, NWB Non-Weight-Bearing, WB Weight-Bearing.

ROM indicated that the best cutoff value for determining the risk of AT was $22^{\circ}$, with a corresponding sensitivity and specificity of $80 \%$ and $86 \%$, respectively, and an area under the curve of 0.80 .

No difference in quality of movement (LSD) was noted between healthy participants and those with AT $(p=0.361)$.

\section{Discussion}

Ankle DF ROM measured with the knee bent was predictive of the development of AT in a sample of 70 military recruits. To the best of our knowledge, this study is the first to implicate this factor in the risk of AT.

The difference between uninjured participants and those that developed AT exceeded the minimal detectable change of the DF measurement that was used in this study $\left(6.2^{\circ}\right)$ [25], thus further supporting the existence of a true DF ROM difference between these 2 groups. The most accurate DF cutoff threshold for predicting AT was $22^{\circ}$, with 4 of the 5 injured participants demonstrating range values below this threshold, compared to only 9 of 65 uninjured participants. Previous investigations suggest that the ankle dorsiflexes approximately $20^{\circ}$ during the stance phase of running $[13,26]$. This means that if the available DF ROM of an athlete was approximately $20^{\circ}$, their ankle would need to be stressed very close to its DF limit with every step during running. Over time, this may lead to injury.

Although its role in the pathogenesis of AT is controversial [9], subtalar pronation may be another mechanism by which limited ankle DF may cause AT. A more limited DF ROM may lead to compensatory subtalar pronation [27] which, in turn, may lead to greater tibial internal rotation. When this occurs during mid- to latestance it may cause "wringing out" of the Achilles tendon due to the simultaneous knee extension which is accompanied by tibial external rotation $[8,14]$. Excessive subtalar pronation may also contribute to the development of AT as it necessitates greater activation of the gastro-soleus complex during running [8].
Unlike NWB DF ROM, the WB measurement did not reveal a significant difference between injured and uninjured participants. Although injured participants did display $6^{0}$ less WB DF, this difference was mainly due to extremely low values among 2 of the 5 injured participants $\left(31.1^{0}\right.$ and $35.7^{\circ}$ ), while the other 3 participants displayed range values that were very close to the sample mean $\left(48.3^{0}\right.$, $50.0^{\circ}$ and $51.0^{\circ}$ degrees). The discordance between WB and NWB DF is not surprising given that either measurement has been shown to account for only $35 \%$ of the variance in the other [25]. Interestingly WB DF ROM on the left side was more limited among injured compared with uninjured participants (Table 2), and a similar trend was noted for left NWB DF ROM. Combined with the findings on the right side, these differences suggest that injured participants tended to display a more limited ankle DF ROM bilaterally. The fact that all injuries still occurred on the right side may be related to differences in the usage of the 2 extremities. For example, it has been previously shown that during gait the power generation of the right lower extremity is more associated with propulsion, while that of the left is more associated with postural control [28]. Consequently, even in the presence of a symmetrically limited DF ROM, the right side may still be more prone to developing AT. The association between sidedness and the development of AT, as well as other overuse lower extremity injuries, may be worth exploring.

Although not statistically significant, a greater proportion of injured participants displayed a faulty lower extremity movement pattern during the LSD test $(80 \%$ among injured participants versus $51 \%$ among uninjured participants). This movement pattern has been previously associated with decreased ankle DF ROM and increased subtalar pronation $[24,29]$. Three of the injured participants displayed a moderate movement pattern (score 3/6) during the LSD test, while a fourth participant, which was subsequently grouped with the "Moderate" quality group, actually displayed a "Poor" pattern based on the LSD 
scoring criteria (score 4/6). Due to the overall small number of injured participants, the possibility of a type 2 error should be considered, and we recommend further testing of the predictive validity of the LSD test in AT. Furthermore, it is possible that with the use of a more quantitative assessment method of joint and/or segment alignment during the LSD, the predictive ability of this test would be enhanced.

Our findings are at odds with 2 previous studies that did not find an association between limited bent-knee ankle DF and AT $[10,11]$. These differences may be partly explained by the way DF was measured. The DF ROM in our study was somewhat larger than that found by these studies $\left(27^{0}\right.$ in our study vs. $20-23^{0}$ previously) $[10,11]$. Mahieu et al. [11] measured DF both actively and passively in a supine (gravity-resisted) position, with the knee bent $45^{\circ}$ [11]. We used a prone (gravity-assisted) position with the knee bent $90^{\circ}$, which may explain the greater ROM found in our study. Although measuring ankle DF ROM with the knee bent approximately $40^{\circ}$ may be more representative of the functional demands during running, we believe the prone $90^{\circ}$ knee flexion position, which places the tibia in a vertical alignment, allows the examiner to use his/her body weight more effectively in order to stretch the ankle fully into its DF limit. In the study by Kaufman et al. [10] neither the position of the measurement, nor whether active or passive motion was measured, were specified [10]. Finally, while no information regarding measurement reliability was provided by these previous investigations, our technique has been previously proven reliable when performed by the examiners involved in this study [24].

Our study has several important limitations. First, the number of cases of AT was low $(n=5)$, leading to a wide 95\% CI around the point value of the predictor. The incidence of AT in our study was $7 \%$, which is similar to that found by Kaufman et al. [10] over a similar followup period (5\%), but lower than that found by Mahieu et al. [11] over a 6-week follow-up period (14\%). As the diagnosis of AT was based on history and physical examination in all studies, we believe differences in training regimens, rather than diagnostic criteria, are more likely to explain the different incidence rates. The lack of use of imaging for the diagnosis of AT could be considered another limitation of this study. However, our diagnostic criteria are compatible with current clinical practice guidelines [8]. Furthermore, recent evidence suggests that pain on palpation of the tendon, and the subjective report of pain between 2 to $6 \mathrm{~cm}$ proximal to the insertion of the tendon, are the most accurate diagnostic criteria for AT [30]. Our findings may also be limited to a relatively young population, undergoing rigorous physical training. Nutritional regimen, hours of sleep, training terrains, loads carried and shoe wear may also differ between a military and a civilian population, thus presenting another possible limitation. Finally, due to a limited number of investigators and participants, we were not able to obtain other possible predictors during baseline testing. Thus, the effects of other variables such as DF ROM with the knee extended, subtalar mobility, endurance or strength of various muscle groups could not be considered.

\section{Conclusions}

Limited ankle DF ROM, as measured in NWB with the knee bent, may increase the risk of developing AT in army recruits taking part in intense physical training. Future studies are needed to further validate limited bent-knee ankle DF as a risk factor for AT, as well as to assess whether a cutoff threshold of $22^{\circ}$ can most accurately predict the occurrence of this condition. Finally, despite its inability to predict AT in this study, we encourage future investigation of the LSD test as a screening tool for the risk of AT.

\section{Abbreviations}

ABT: Army basic training; AT: Achilles tendinopathy; DF: Dorsiflexion: LSD: Lateral step down; NWB: Non-weight-bearing; OR: Odds ratio; ROC: Receiver operator curve; ROM: Range of motion; WB: Weight-bearing.

\section{Competing interests}

The authors declare that they have no competing interests.

\section{Authors' contributions}

AR participated in the design, data collection, statistical analysis and manuscript preparation. ZK participated in the design of the study, data collection, and manuscript revising. ASF conceived the study, participated in data collection and manuscript revising. All authors read and approved the final manuscript.

\section{Author details}

${ }^{1}$ Department of Physiotherapy, Ariel University, Ariel, Israel. ${ }^{2}$ Department of Orthopaedic Surgery, Assaf Harofeh Medical Center, Zerrifin, Israel. ${ }^{3}$ Sackler School of Medicine, Tel-Aviv University, Tel-Aviv, Israel. ${ }^{4}$ Israel Defense Force Medical Corps, Haifa, Israel.

Received: 27 August 2014 Accepted: 31 October 2014

Published online: 18 November 2014

\section{References}

1. de Jonge S, van den Berg C, de Vos RJ, van der Heide HJ, Weir A, Verhaar JA, Bierma-Zeinstra SM, Tol JL: Incidence of midportion Achilles tendinopathy in the general population. Br J Sports Med 2011, 45(13):1026-1028.

2. Jacobsson J, Timpka T, Kowalski J, Nilsson S, Ekberg J, Renström P: Prevalence of musculoskeletal injuries in Swedish elite track and field athletes. Am J Sports Med 2012, 40(1):163-169.

3. Lopes AD, Hespanhol JLC, Yeung SS, Costa LO: What are the main running-related musculoskeletal injuries? A systematic review. Sports Med 2012, 42(10):891-905.

4. Sankey RA, Brooks JH, Kemp SP, Haddad FS: The epidemiology of ankle injuries in professional rugby union players. Am J Sports Med 2008, 36(12):2415-2424.

5. Tenforde AS, Sayres LC, McCurdy ML, Collado H, Sainani KL, Fredericson M: Overuse injuries in high school runners: lifetime prevalence and prevention strategies. PM R 2011, 3(2):125-131.

6. Heir T: Musculoskeletal injuries in officer training: one-year follow-up. Mil Med 1998, 163(4):229-233. 
7. Milgrom C, Finestone A, Zin D, Mandel D, Novack V: Cold weather training: a risk factor for Achilles paratenditnits among recruits. Foot Ankle Int 2003, 24(5):398-401.

8. Carcia CR, Martin RL, Houck J, Wukich DK: Achilles pain, stiffness, and muscle power deficits: achilles tendinitis. J Orthop Sports Phys Ther 2010, 40(9):A1-A26.

9. Munteanu SE, Barton CJ: Lower limb biomechanics during running in individuals with achilles tendinopathy: a systematic review. J Foot Ankle Res 2011, 4:15

10. Kaufman KR, Brodine SK, Shaffer RA, Johnson CW, Cullison CR: The effect of foot structure and range of motion on musculoskeletal overuse injuries. Am J Sports Med 1999, 27(5):585-593.

11. Mahieu NN, Withvrouw E, Stevens V, Van Tiggelen D, Roget P: Intrinsic risk factors for the development of achilles tendon overuse injury: a prospective study. Am J Sports Med 2006, 34(2):226-235.

12. Novacheck TF: The biomechanics of running. Gait Posture 1998, 7(1):77-95.

13. Donoghue OA, Harrison AJ, Laxton P, Jones RK: Lower limb kinematic of participants with chronic achilles tendon injury during running. Res Sports Med 2008, 16(1):23-38

14. Schepsis AA, Jones $H$, Haas AL: Achilles tendon disorders in athletes. Am J Sports Med 2002, 30(2):287-305.

15. Sasaky K, Neptune RR: Differences in muscle function during walking and running at the same speed. J Biomech 2006, 39(11):2005-2013.

16. You JY, Lee HM, Luo HJ, Leu CC, Wu SK, Cheng PG: Gastrocnemius tightness on joint angle and work of lower extremity during gait. Clin Biomech (Bristol, Avon) 2009, 24(9):744-750.

17. Kulig K, Loudon JK, Popovich JM, Pollard CD, Winder BR: Dancers with achilles tendinopathy demonstrate altered lower extremity takeoff kinematics. J Orthop Sports Phys Ther 2011, 41(8):606-613.

18. Powers CM: The influence of altered lower-extremity kinematics on patellofemoral joint dysfunction: a theoretical perspective. J Orthop Sports Phys Ther 2003, 33(11):639-646.

19. Salsich GB, Graci V, Maxam DE: The effects of movement pattern modification on lower extremity kinematics and pain in women with patellofemoral pain. J Orthop Sports Phys Ther 2012, 42(12):1017-1024

20. Boling MC, Padua DA, Marshall SW, Guskiewicz K, Pyne S, Beutler A: A prospective investigation of biomechanical risk factors for patellofemoral pain syndrome. The joint undertaking to monitor and prevent $\mathrm{ACL}$ injury (JUMP-ACL) cohort. Am J Sports Med 2009, 37(11):2108-2116.

21. Hewett TE, Myer GD, Ford KR, Heidt RS Jr, Colosimo AJ, McLean SG, van den Bogert AJ, Paterno MV, Succop P: Biomechanical measures of neuromuscular control and valgus loading of the knee predict anterior cruciate ligament injury risk in female athletes: a prospective study. Am J Sports Med 2005, 33(4):492-501.

22. Lowry CD, Cleland JA, Dyke K: Management of patients with patellofemoral pain syndrome using a multimodal approach: a case series. J Orthop Sports Phys Ther 2008, 38(11):691-702.

23. Piva SR, Fitzgerald K, Irrgang JJ, Jones S, Hando BR, Browder DA, Childs JD: Reliability of measures of impairments associated with patellofemoral pain syndrome. BMC Musculoskelet Disord 2006, 7:33

24. Rabin A, Kozol Z: Measures of range of motion and strength among healthy women with differing quality of lower extremity movement during the lateral step-down test. J Orthop Sports Phys Ther 2010, 40(12):792-800.

25. Rabin A, Kozol Z: Weightbearing and nonweightbearing ankle dorsiflexion range of motion: are we measuring the same thing? J Am Podiatr Med Assoc 2012, 102(5):406-411.

26. Hamner SR, Seth A, Delp SL: Muscle contributions to propulsion and support during running. J Biomech 2010, 43(14):2709-2716.

27. Gross T: Lower quarter screening for skeletal malalignment - suggestions for orthotics and shoewear. J Orthop Sports Phys Ther 1995, 21(6):389-405.

28. Sadeghi H, Allard P, Duhaime M: Contributions of lower-limb muscle power in gait of people without impairments. Phys Ther 2000, 80(12):1188-1196.
29. Joseph M, Tiberio D, Baird JL, Trojian TH, Anderson JM, Kraemer WJ, Maresh CM: Knee valgus during drop jumps in National Collegiate Athletic Association Division I female athletes: the effect of a medial post. Am J Sports Med 2008, 36(2):285-289.

30. Hutchinson AM, Evans R, Bodger O, Pallister I, Topliss C, Williams P, Vannet $\mathrm{N}$, Morris $\mathrm{V}$, Beard D: What is the best clinical test for Achilles tendinopathy? J Foot Ankle Surg 2013, 19(2):112-117.

doi:10.1186/s13047-014-0048-3

Cite this article as: Rabin et al: Limited ankle dorsiflexion increases the risk for mid-portion Achilles tendinopathy in infantry recruits: a prospective cohort study. Journal of Foot and Ankle Research 2014 7:48.

\section{Submit your next manuscript to BioMed Central and take full advantage of:}

- Convenient online submission

- Thorough peer review

- No space constraints or color figure charges

- Immediate publication on acceptance

- Inclusion in PubMed, CAS, Scopus and Google Scholar

- Research which is freely available for redistribution

Submit your manuscript at www.biomedcentral.com/submit
C BioMed Central 